\title{
Contexto de uso: Conceito, fatores e modelos
}

\section{Context of use: Concept, factors and models}

MACHADO, Laís; Mestra; Universidade Federal de Santa Catarina - UFSC

I.machado@posgrad.ufsc.br

VERGARA, Lizandra Garcia Lupi Vergara; Doutora; Universidade Federal de Santa Catarina - UFSC

I.vergara@ufsc.br

\section{Resumo}

O presente artigo, relata os fatores envolvidos no contexto de uso, quando se trata de dispositivos móveis. O estudo consiste em um levantamento da literatura, por meio de uma pesquisa bibliográfica em artigos, livros, teses e dissertações, elucidando os conceitos do contexto de uso, pela perspectiva de diferentes autores. Assim, a partir dos resultados, é possível ter um entendimento amplo dos elementos que interferem a experiência do usuário, no uso dos dispositivos móveis.

Palavras Chave: Contexto de uso; usabilidade; interação humano-computador (IHC).

\begin{abstract}
This article reports on the factors involved in the context of use when it comes to mobile devices. The study consists of a survey of the literature, through a bibliographical research, in articles, books, theses and dissertations, elucidating the concepts of the context of use, from the perspective of different authors. Thus, from the results, it is possible to have a broad understanding of the elements that interfere with the user experience in the use of mobile devices.
\end{abstract}

Keywords: Contexto f use; usability; human-computer interaction ( $\mathrm{HCl})$.

\section{Introdução}

Há um crescente interesse tanto da indústria quanto da academia, no desenvolvimento de produtos com uma boa experiência. Este fato, tem proporcionado grandes avanços na área de design, usabilidade e experiência do usuário (UX).

Do mesmo modo, no desenvolvimento de interfaces, há uma busca por soluções que proporcionem experiência envolvente ao usuário; maior satisfação no uso, identificação do usuário com aspectos visuais, formas de interação agradáveis, entre outros. No entanto, em projetos com foco nos dispositivos móveis, além dos fatores ligados a experiência, deve-se estar atendo as interferências do ambiente, pois podem afetar diretamente a experiência nestes dispositivos.

Maguire (2001) relata que a usabilidade de um produto ou sistema, pode ser alterada de acordo com seu contexto de uso. Por definição, o contexto de uso são todos os elementos que podem influenciar a forma como o usuário irá interagir com os objetos, produtos ou sistemas. Se trata dos fatores externos a interface do produto, porém que interferem diretamente a interação. (BARNARD 
et al. 2007; MAGUIRE, 2001; PEIXOTO, 2017).

Quando se trata de dispositivos móveis, o contexto de uso, é um desafio para os desenvolvedores que visam uma boa experiência dos produtos. Estes dispositivos podem ser utilizados em inúmeras possibilidades de contexto, de intensidade luminosa, ruídos, intempéries, interações com outras pessoas entre outros fatores. (ROTO VIRPE et al., 2006; PREZOTTO, BONIATI, 2017).

O presente estudo tem como objetivo auxiliar o processo de compreensão entre os conceitos de ergonomia, usabilidade e contexto de uso. Para isto, realiza um levantamento na literatura acerca dos fatores intervenientes do contexto de uso. Tais fatores, podem afetar diretamente a experiência do usuário na interação com dispositivos móveis.

Destaca-se que o presente estudo é parte de um projeto de tese, no qual possui seu foco nas relações entre a experiência do usuário (User Experience - UX) e os fatores envolvidos no contexto de uso. Assim, os conceitos, discussões e resultados deste artigo, servirão de subsídio para o autor deste estudo, assim como podem dar suporte para outros autores que tenham pesquisa na mesma área em questão.

\section{Procedimentos Metodológicos}

No presente tópico estão dispostos: a caracterização da pesquisa, quanto aos seus objetivos, procedimentos, abordagem e sua natureza; e as etapas e procedimentos realizados no presente estudo.

\subsection{Caracterização da pesquisa}

A presente pesquisa se caracteriza, quanto aos seus objetivos, como um estudo exploratório, no qual a partir da literatura apresenta os conceitos e busca uma maior familiaridade com o tema. Pela perspectiva dos procedimentos de coleta, se caracteriza como pesquisa bibliográfica, elaborada a partir de material já publicado em livros, revistas, artigos, dissertações, teses entre outros. Sobre sua abordagem, se classifica como qualitativa à medida que se baseia em ideias teóricas e no conteúdo publicado. Quanto a natureza, se define como uma pesquisa básica busca novos conhecimentos (LAKATOS e MARCONI, 2011).

\subsection{Etapas da pesquisa}

O estudo se dividiu em duas etapas: (I) Levantamento bibliográfico e (II) Compreensão e análise. Primeiramente, realizou-se o levantamento dos conceitos relacionados ao tema pela perspectiva de diferentes autores (etapa I). Este levantamento foi realizado com base na literatura científica atual, em artigos, livros, revistas científicas, teses e dissertações. Em um segundo momento realizou-se as análises e compreensão das relações entre os conceitos apresentados (etapa II).

\section{Relações entre Ergonomia, usabilidade e contexto de uso}

A ergonomia se baseia em um conjunto de conhecimentos científicos relacionados ao homem que são necessários para construir e conceber instrumentos, equipamentos e dispositivos para serem usados com o máximo de conforto, segurança e eficácia por seus usuários. Não se restringe apenas à adaptação do homem às máquinas e equipamentos, mas em todo o processo que ocorre durante o relacionamento do homem e seu trabalho (WISNER, 1987; IIDA, 1990).

Como ciência a ergonomia desenvolve conhecimentos sobre as capacidades, limites e outras 
características do desempenho humano, que se relacionam com o projeto de interfaces, entre indivíduos e outros componentes do sistema. Como prática a ergonomia compreende a aplicação de tecnologia de interface homem-sistema a projeto ou modificações de sistemas para aumentar a segurança, conforto e eficiência do sistema e de qualidade de vida (MORAES, 2000; MORAIS, SEABRA, MATTEDI, 2018).

Segundo a ISO 9241-210 (2010) "A ergonomia é o estudo dos fatores humanos, disciplina cientifica concentrada em compreender as interações entre o homem e outros elementos de um sistema, e o profissional que aplica a teoria, princípios, dados e métodos para projetar, a fim de otimizar o bem-estar humano e o desempenho geral de um sistema"(ISO 9241-210, 2010; REMUSZKA, OLIVEIRA, 2017).

A ergonomia está na origem da usabilidade, pois ela visa proporcionar a eficiência e eficácia, além do bem-estar e saúde do usuário, por meio da adaptação do trabalho ao homem. Assim, a ergonomia pretende garantir que sistemas e dispositivos estejam adequados à maneira do usuário pensar, se comportar, trabalhar, proporcionando conforto. (CYBIS, 2010)

Shackel (1986) apresenta que o conceito de usabilidade começou a ser aplicado a partir da década de 1970, com o desenvolvimento e avaliação dos softwares. A partir de 1990 o conceito de usabilidade foi ampliado e aplicado aos produtos de consumo. Neste período não havia muita diferenciação nos fatores avaliados na usabilidade de software e de outros produtos. Porém ao longo do tempo, percebeu-se, que diferente das interfaces, nos produtos os aspectos subjetivos da usabilidade são tão importantes quanto o seu desempenho.

O IHC, (Interação Humano-Computador) é um campo de estudo que busca compreender as relações entre o homem e o uso, entendimento ou a ausência de entendimento da tecnologia da informação. É uma disciplina que busca estabelecer a forma como as pessoas interagem com os computadores em um contexto organizacional (TE'ENI et al., 2007). Pode ser definido também como o estudo dos fatores humanos e os aspectos da máquina em processo de interação (ALSHEHRI e FREEMAN, 2012).

Para Cañas e Waerns (2001), ao se falar em interface deve-se incluir o meio pelo qual o artefato apresenta a informação à pessoa e o meio pelo qual a pessoa insere informação no artefato. Assim, para o usuário, a interface é o meio em que se opera a interação, que permite estabelecer um contato físico, perceptivo e conceitual entre a pessoa e o artefato tecnológico.

Outros autores, (ALSHEHRI e FREEMAN, 2012) relatam que a pesquisa $\mathrm{HCl}$ pode ampliar a compreensão acerca da eficiência da interface alcançada por meio de alta qualidade no design de interface, que não tenha apenas um bom desempenho, mas que tenha uma preocupação e incorpore os elementos humanos tais como experiência físicas e sociais da interação.

Segundo Moraes (1996), os problemas de interface podem ser categorizados como problemas de utilidade, informacionais ou problemas de usabilidade. Os de utilidade estão relacionados ao impedimento do usuário em realizar a tarefa desejada. Já os informacionais estão mais ligados à diagramação, ao modo de agrupamento das informações e legibilidade. Os problemas de usabilidade estão ligados à interação humano-computador de forma que o sistema impede que o usuário realize seus objetivos na interface.

Quanto a usabilidade, tem-se como uma das definições mais comuns a proposta pela ISO/IEC 9241(2000): "usabilidade é um conjunto de atributos de software relacionado ao esforço necessário para seu uso e para o julgamento individual de tal uso por determinado conjunto de usuários". Para a International Organization for Standardization (para o português Organização Internacional para Padronização) e de acordo com a ISO 9241:11 (2002), a usabilidade é uma referência de quanto 
um usuário específico pode utilizar um produto específico para alcançar objetivos específicos, tendo eficácia, eficiência e satisfação.

A ISO 9241-11 (2002) faz algumas definições acerca da usabilidade como:

- Eficácia: Acurácia e completude com as quais usuários alcançam objetivos específicos.

- Eficiência: Recursos gastos em relação à acurácia e abrangência com as quais usuários atingem objetivos.

- Satisfação: Ausência do desconforto e atitudes positivas para com o uso de um produto.

- Contexto de uso: Usuários, tarefas, equipamento (hardware, software e materiais), e o ambiente físico e social no qual um produto é usado.

- Sistema de trabalho: Sistema, composto de usuários, equipamento, tarefas e o ambiente físico e social, com o propósito de alcançar objetivos específicos.

- Usuário: Pessoa que interage com o produto.

- Objetivo: Resultado pretendido.

- Tarefa: Conjunto de ações necessárias para alcançar um objetivo.

- Produto: Parte do equipamento (hardware, software e materiais) para o qual a usabilidade é especificada ou avaliada.

A ISO/IEC 9126-1(2001) identifica a usabilidade como sendo um dos seis atributos de qualidade de software diferentes. O padrão ISO 9251-11(1998), define usabilidade como eficiência, eficácia e satisfação dos usuários nos seus objetivos na interface. Há também uma diretiva 90/270/CEE do Conselho da União Européia (1990) que trata sobre requisitos mínimos de segurança e saúde para o trabalho de computadores (SEFFAH, et al, 2006; BEVAN, 2008; MORAIS, SEABRA, MATTEDI, 2018).

Usabilidade é um atributo qualitativo que indica a facilidade ou dificuldade de uma interface para os usuários, também utilizado para referenciar método. Os problemas quanto à usabilidade podem trazer consequências para a interação e devem ser estudados a fim de evitá-los em projetos de interface. Esses problemas podem ser classificados como de barreira, obstáculo e ruído. (CYBIS, 2010). SEFFAH, et al, (2006) realizou um mapeamento dos atributos de usabilidade de vários padrões e modelos, obtendo a tabela a seguir:

Quadro 1 - atributos de usabilidade de vários padrões e modelos

\begin{tabular}{|c|c|c|c|c|c|}
\hline $\begin{array}{l}\text { Constantine \& } \\
\text { Lockwood } \\
\text { (1999) }\end{array}$ & $\begin{array}{l}\text { ISO 9241-11 } \\
(1998)\end{array}$ & $\begin{array}{c}\text { Schneiderman } \\
\text { (1992) }\end{array}$ & $\begin{array}{l}\text { Nielsen } \\
\text { (1993) }\end{array}$ & $\begin{array}{l}\text { Preece et al } \\
(1994)\end{array}$ & $\begin{array}{l}\text { Shackel } \\
(1991)\end{array}$ \\
\hline $\begin{array}{c}\text { Eficiência no } \\
\text { uso }\end{array}$ & Eficiência & $\begin{array}{l}\text { Velocidade da } \\
\text { performance }\end{array}$ & $\begin{array}{c}\text { Eficiência no } \\
\text { uso }\end{array}$ & $\begin{array}{c}\text { Taxa de } \\
\text { transferência }\end{array}$ & $\begin{array}{c}\text { Eficácia } \\
\text { (velocidade) }\end{array}$ \\
\hline $\begin{array}{l}\text { Capacidade de } \\
\text { aprendizado }\end{array}$ & & $\begin{array}{c}\text { Tempo de } \\
\text { aprendizado }\end{array}$ & $\begin{array}{l}\text { Aprendizado } \\
\text { (facilidade de } \\
\text { aprendizado) }\end{array}$ & $\begin{array}{l}\text { Aprendizado } \\
\text { (facilidade de } \\
\text { aprendizado) }\end{array}$ & $\begin{array}{l}\text { Aprendizado } \\
\text { (tempo de } \\
\text { aprendizagem) }\end{array}$ \\
\hline Lembrança & & $\begin{array}{l}\text { Retenção sobre } \\
\text { o tempo }\end{array}$ & Memorização & & $\begin{array}{l}\text { Aprendizado } \\
\text { (retenção) }\end{array}$ \\
\hline $\begin{array}{l}\text { Confiabilidade } \\
\text { no uso }\end{array}$ & & $\begin{array}{l}\text { Taxa de erros } \\
\text { pelos usuários }\end{array}$ & $\begin{array}{c}\text { Erros/ } \\
\text { Segurança }\end{array}$ & $\begin{array}{c}\text { Taxa de } \\
\text { transferência }\end{array}$ & Eficácia (erros) \\
\hline $\begin{array}{l}\text { Satisfação do } \\
\text { usuário }\end{array}$ & $\begin{array}{l}\text { Satisfação (conforto e } \\
\text { aceitabilidade no uso) }\end{array}$ & $\begin{array}{l}\text { Satisfação } \\
\text { subjetiva }\end{array}$ & Satisfação & Atitude & Atitude \\
\hline
\end{tabular}

Fonte: SEFFAH, et al, (2006) traduzido pelo autor 
Seffah, et, al. (2006) afirmam que usabilidade foi definida de diferentes maneiras o que torna o conceito confuso. Isto posto, o autor apresenta alguns diferentes padrões: "Um conjunto de atributos que dependem do esforço necessário para o uso e da avaliação individual de tal uso por um conjunto de usuários declarado um implícito" (ISO/IEC 9126-1991). "A medida em que um produto pode ser usado por usuários específicos para atingir metas específicas com eficácia, eficiência e satisfação em um contexto específico de uso" (ISO 9241-11, 1998). "A facilidade com que um usuário pode aprender a operar, preparar entradas e interpretar saídas, de um sistema ou componente" (IEEE Std.610.12-1990).

Dentro do mesmo contexto, o autor Seffah, et al (2006) estabelece a existência de três grandes padrões ISO / IEC para quantificar e mensurar usabilidade. O primeiro sendo o padrão ISO 9241-11 (1998) que identifica eficiência, eficácia e satisfação como atributos principais de usabilidade. Por segundo o padrão ISO / IEC 9136-1 (2001) no qual apresenta usabilidade como sendo um atributo de qualidade de software, que pode ser decomposto em cinco fatores, sendo estes: compreensão, aprendizagem, operabilidade, atratividade e compatibilidade da usabilidade com guias de estilo já publicados. E o último, o padrão ISO / IEC 9126-4 (2001) definindo o conceito relacionado de qualidade em uso como um tipo de atributo de qualidade de software de ordem superior, decomposta em três fatores: eficácia, produtividade e segurança (SEFFAH, et al., 2006).

Para o autor, o padrão ISO 9241-11 (1998), possui uma perspectiva mais ampla que outros padrões mais recentes. Enquanto o padrão antigo concentra-se em questões da tarefa e meio ambiente, suas definições de usabilidade referem-se a características de qualidade de software, os mais recentes como os padrões ISO 9126, se concentram na funcionalidade, precisão e eficácia. (BEVAN, 2008; SEFFAH, et al., 2006; MORAIS, SEABRA, MATTEDI, 2018).

Os problemas de barreira são dificuldades na interface que impedem a realização da tarefa pelo usuário. A barreira pode ser qualquer função temporária ou definitiva que não permite que o usuário, por mau entendimento ou por não possuir algum conhecimento técnico específico, alcance seu objetivo na interface. A presença desse tipo de impedimento implica em prejuízos tanto para os usuários quanto para os projetistas que desenvolvem uma função que não está sendo utilizada.

Os problemas de obstáculo referem-se a um aspecto da interface que o usuário não compreende, porém não chega a impedir o uso do sistema. Os problemas de obstáculo prejudicam o entendimento e a eficácia da função em questão.

Já os problemas de ruído são dificuldades que não se enquadram em barreira ou obstáculo, mas causam alguma diminuição do desempenho da interface. A principal característica do ruído é que pode causar uma má impressão ao usuário do sistema (aspecto subjetivo). Pode ser identificado como uma pergunta mau formulada, que faça o usuário pensar excessivamente (CYBIS, 2010) (ver figura 1). 
Figura 1 - Problemas de usabilidade

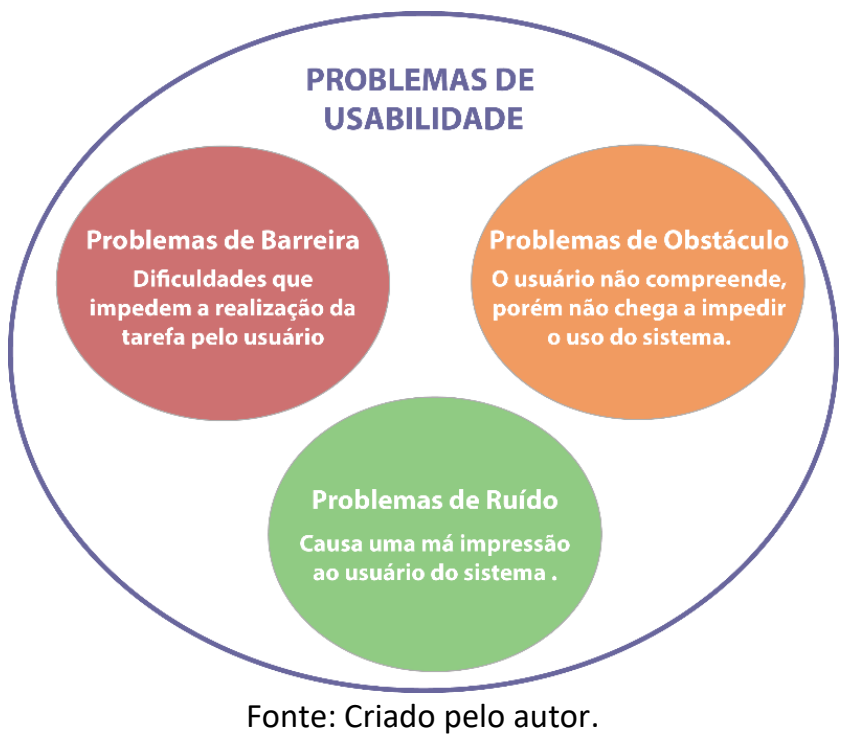

Cybis (2010) ainda apresenta os problemas de usabilidade relacionados ao tipo de usuário que o problema de usabilidade afeta. Pode ser o público em geral que é relacionado a um aspecto da interface que irá atrapalhar qualquer tipo de usuário que utilizar a interface. Afetar um público especializado, na qual irá afetar apenas usuários que já são especialistas no sistema e já o utilizam com experiência. De intuitividade, no qual o tipo de problema de usabilidade que atrapalha mais especificamente usuários novatos. E de acessibilidade, que são problemas que afetam usuários em condições especiais (portadores de deficiência, por exemplo), passando despercebidos de usuários comuns. (CYBIS, 2010).

Os termos usabilidade e experiência do usuário são muito usados na literatura como sinônimos, porém existem grandes distinções entre esses termos. De acordo com Alshehri e Freeman (2012) a usabilidade é um conceito mais restrito em relação à experiência do usuário, visto que a usabilidade verifica apenas a facilidade do uso, enquanto a experiência do usuário inclui sentimentos do usuário sobre a interação.

\section{Contexto de uso}

Os dispositivos móveis são projetados para serem portáteis, isso significa que estes produtos podem ser utilizados em diferentes ambientes e situações de uso, como utilizá-lo tanto a luz quanto no escuro, em ambientes abertos e fechados, em ambientes com ruídos ou silenciosos. (ROTO VIRPE et al., 2006).

O contexto de uso, para Barnard, et al. (2007), é um conjunto de condições que podem influenciar nas maneiras pelas quais um ser humano interage com um dispositivo de computação móvel (BARNARD, et al.,2007; PEIXOTO, 2017).

A ISO 9241-11 (1998) apresenta o conceito de contexto de uso relacionado ao desenvolvimento de software como sendo as tarefas, os usuários, os equipamentos, (hardware, software e materiais) o ambiente físico e social no qual o produto está sendo utilizado.

Ainda tratando sobre os dispositivos móveis, Bertini et al. (2009), relata que ao se tratar de limitações do uso de dispositivos móveis, tem-se duas categorias: limitações do dispositivo e limitações do contexto. 
As limitações do dispositivo são:

- Tamanho pequeno da tela: Para promover a portabilidade dos dispositivos, estes são desenvolvidos com tamanhos reduzidos o que pode prejudicar a usabilidade do produto.

- Entrada de dados limitada: Devido ao formato dos dispositivos, os mecanismos de entrada de dados podem ser limitados.

- Custo e quantidade de banda limitados: Fatores que limitam as conexões de internet. Podem ser acessados

- Limitação de conectividade: Latência da conexão variável

- Limitação computacional e recursos: Capacidades das aplicações são limitadas

- Limitação de bateria: As baterias ainda são um grande problema para qualquer dispositivo móvel. Isso tem um grande impacto no usuário final que possui autonomia limitada, disponibilidade limitada.

- Ampla heterogeneidade: Mudança de dispositivo físico e operacional, reaprendendo as funções e operações.

Sob o mesmo foco, sobre as limitações de uso, Bertini et al. (2009) relatam cinco fatores do contexto que podem interferir na interação:

- Contexto variável: $\mathrm{O}$ contexto que os usuários utilizarão o dispositivo é variável e estará em alteração o tempo todo;

- Tipo de interação: A natureza da interação também muda quando se trata de dispositivos móveis. A realização de tarefas em dispositivos móveis consiste em pequenos e rápidos passos e devem ser executados sem exigir muito esforço cognitivo. Assim, as tarefas devem ser realizadas em condições que a atenção do usuário pode estar reduzida.

- Interrupções: Notificações e perguntas podem acontecer em momentos inapropriados, podendo interromper alguma tarefa que está sendo realizada.

- Privacidade e segurança: No uso dos dispositivos móveis o usuário pode ser encontrar em diferentes lugares, (tanto privados como públicos) e em uma variedade de situações (formais ou informais e também com diferentes infraestruturas (com wireless ou conectividade a cabo). Assim, estas diferentes configurações conferem diferentes necessidades.

- Intimidade e disponibilidade: estes dispositivos são compreendidos como pessoais, tendo o usuário uma relação diferente entre um PC e um Smartphone.

Para Mallat et al (2009) as tecnologias móveis mudaram o modo de operação dos usuários, estes começaram a carregar os dispositivos em seus bolsos ou em bolsas, mochilas e assim usá-los em qualquer lugar. Consequentemente, apareceram alguns problemas dependendo do local que era utilizado. No entanto, a mobilidade que garantiu a grande aceitação dos dispositivos móveis.

\subsection{Modelos de contexto de uso}

No início dos estudos do contexto, Belk (1975) definiu cinco categorias do ambiente:

a) Físico: incluindo localização geográfica e institucional, sons, vistas e o tempo;

b) Social: definido pela interação interpessoal e a influência de outras;

c) Temporal: consistindo de um ponto no tempo ou o tempo relativo a algum evento passado ou futuro;

d) Definição da tarefa: especificando a função necessária e a que é utilizado;

e) Estados antecedentes: em consequência do humor do usuário, ou possíveis mudanças 
Figura 2 - Contexto Belk (1975)

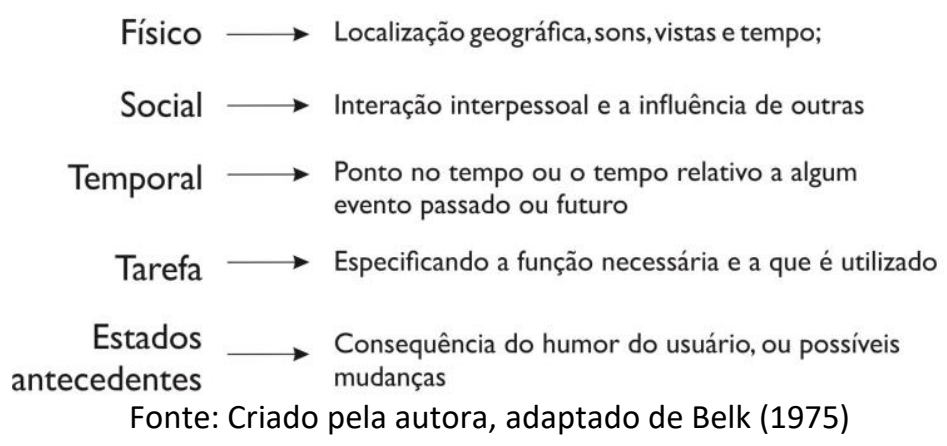

Em estudos posteriores, como de Kim et al (2005), apresentam o contexto de uso dividido em contexto pessoal e contexto do ambiente. O contexto pessoal se divide em interno, no qual inclui objetivos e emoção. Enquanto o contexto externo são as mãos, pernas por exemplo. Enquanto o contexto ambiental inclui o contexto físico (visual e ruído) contexto social (localização e interação com outras pessoas).

Figura 3 - Modelo contexto de Kim et al (2005)

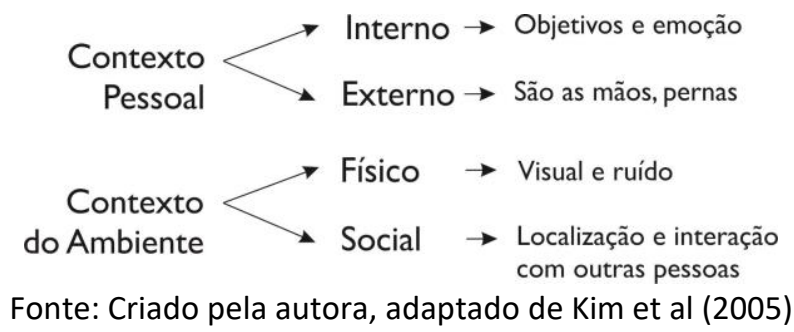

Outros autores, Schmidt, Beigl e Gellersen (1999) apresentam um modelo distinto sobre o mesmo tema. Este modelo apresenta duas segmentações: fatores humanos e físicos do ambiente. Sendo que, os fatores humanos estão ligados ao usuário (conhecimento, estado emocional, condições biofisiológicas), o ambiente social (grupo, interação com outras pessoas), e a tarefa que está sendo realizada (engajamento na tarefa, objetivos).

No ambiente físico, os autores apresentam três categorias: localização (posição absoluta ou relativa do usuário), infraestrutura (recursos computacionais no ambiente, comunicação, performance) e condições físicas do ambiente (barulho, luminosidade disponível, pressão entre outros).

Figura 4 - Modelo contexto de Schmidt, Beigl, e Gellersen (1999)

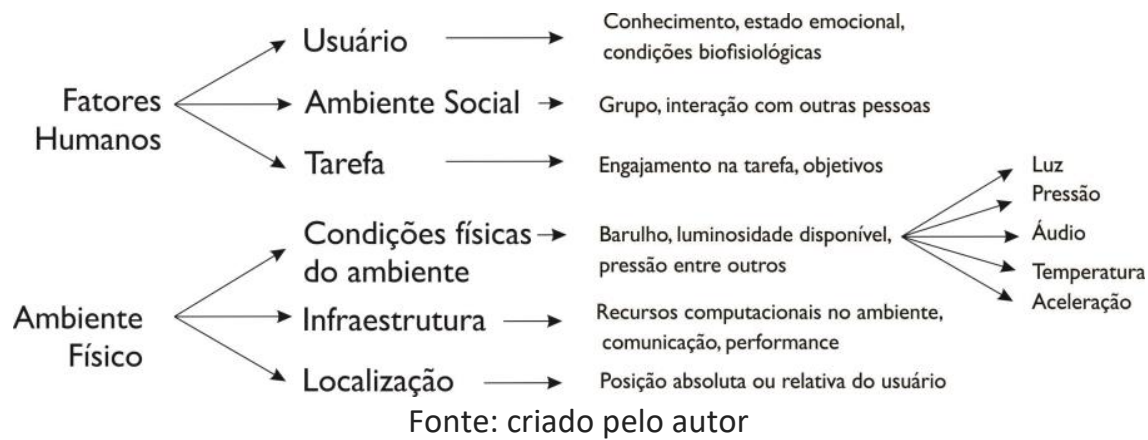


Estas definições de modelos do contexto, sob a ótica de diferentes autores como Belk (1975); Schmidt, Beigl, e Gellersen (1999) e Kim et al (2005), são de grande importância para o presente artigo. Estes fatores apresentados podem dar suporte a pesquisas na área de usabilidade e experiência de uso para dispositivos móveis.

De fato, as definições de contexto, limitações e também modelos dos fatores envolvidos são de grande relevância para o entendimento do contexto de uso. Porém, para Mosqueira-Rey, AlonsoRíos, e Moret-Bonillo (2009), é crucial para a compreensão do contexto de uso de forma profunda, a taxonomia detalhada destes fatores. A taxonomia segundo os autores (MOSQUEIRA-REY ALONSORÍOS e MORET-BONILLO, 2009), lista e define de forma abrangente os atributos do contexto de uso. Ressalta-se que as limitações do espaço não permitem descrever completamente todos os atributos do contexto em detalhes.

Figura 5 - Dimensões do contexto de uso

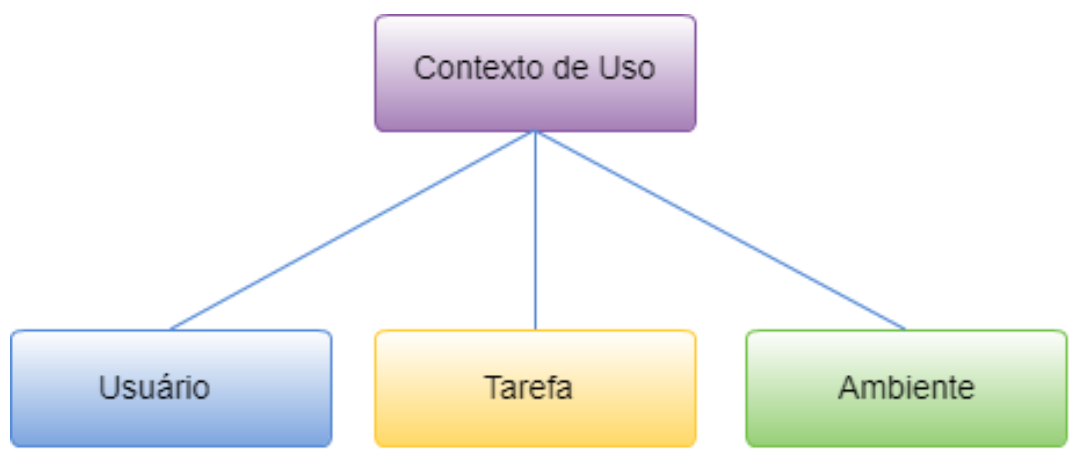

Fonte: Alonso-Ríos, et al. (2010), adaptado pelo autor

Os autores Mosqueira-Rey Alonso-Ríos, e Moret-Bonillo (2009) apresentam uma proposta de taxonomia dos atributos do contexto de uso, organizados em três grupos: usuário, tarefa e ambiente. O grupo chamado usuário possui os seguintes atributos: função, experiência educação, atitude para o sistema, características físicas e características cognitivas. Os atributos citados pelos autores podem ser observados nos quadros (ver quadro 2). 
Quadro 2 - atributos do contexto de uso - Usuário

\begin{tabular}{|c|c|}
\hline \multicolumn{2}{|r|}{ Usuário } \\
\hline Função & $\begin{array}{l}\text { Como funciona a interação do usuário com o sistema, seja em um papel direto (operacional) ou em } \\
\text { um papel indireto, (como um usuário afetado pela interação de outro usuário com o sistema), em } \\
\text { um papel de suporte (realização de manutenção ou instalação tarefas, etc.) ou em uma função de } \\
\text { monitoramento (supervisionando o trabalho dos usuários do sistema). }\end{array}$ \\
\hline Experiência & $\begin{array}{l}\text { Refere-se às habilidades práticas e ao conhecimento do usuário em relação ao sistema, com } \\
\text { distinção entre a experiência com o sistema e experiência com sistemas similares. }\end{array}$ \\
\hline Educação & $\begin{array}{l}\text { É o conhecimento que o usuário adquiriu não através do uso do sistema, mas através da educação } \\
\text { formal, social, cultural ou organizacional. Também o domínio do sistema e a familiaridade com o } \\
\text { qual o sistema pertence. Conhecimento de linguagem do sistema refere-se à capacidade do usuário } \\
\text { de compreender o sistema linguístico de comunicação usada pelo sistema. Conhecimento da } \\
\text { cultura do sistema é a capacidade do usuário de entender a cultura convenções utilizadas pelo } \\
\text { sistema. }\end{array}$ \\
\hline $\begin{array}{l}\text { Atitude para o } \\
\text { sistema }\end{array}$ & Se refere aos sentimentos ou emoções experimentadas pelo usuário ao operar o sistema. \\
\hline $\begin{array}{l}\text { Características } \\
\text { físicas }\end{array}$ & $\begin{array}{l}\text { Características físicas descrevem as características de o corpo do usuário que tem um impacto na } \\
\text { usabilidade, nomeadamente, características sensoriais, características de fala, e características do } \\
\text { motor. Cada característica é descrita em termos de deficiência (limitações funcionais do usuário) e } \\
\text { aptidões (desempenho ótimo de funções do usuário). Ao listar essas distinções, a taxonomia reflete } \\
\text { situações específicas nas quais a mesma característica física apresenta simultaneamente uma } \\
\text { deficiência e uma aptidão. }\end{array}$ \\
\hline $\begin{array}{l}\text { Características } \\
\text { cognitivas }\end{array}$ & $\begin{array}{l}\text { Características cognitivas refere-se à mentalidade características do usuário, com distinção } \\
\text { também desenhada entre deficiências e aptidões. }\end{array}$ \\
\hline
\end{tabular}

Ainda segundo Mosqueira-Rey Alonso-Ríos, e Moret-Bonillo, (2009), há um segundo grupo de atributos do contexto denominado tarefa. Neste grupo o autor apresenta atributos como: escolha no uso do sistema, complexidade, características temporais, demandas, fluxo de trabalho, segurança e criticidade. Todos estes atributos estão especificados no quadro 3.

Quadro 3 - Dimensões do contexto de uso - Tarefa

\section{Tarefa}

Escolha no uso A escolha no uso do sistema pode ser dividida em: duração e frequência. Duração é a medida em do sistema que os usuários podem escolhe se deseja ou não usar o sistema para completara tarefa. E frequência refere-se a como um atributo inclui ambos duração da tarefa e frequência da tarefa

Demanda A demanda possui dois atributos: recursos humanos e recursos materiais. No qual recursos humanos refere-se a subdividido em exigências físicas e demandas congênitas) e solicita material.

Ambiente de Refere-se à extensão a tarefa pode ser controlada pelo usuário durante implementação, trabalho e organizado em desempenho/liberdade e reversibilidade. Desempenho/liberdade se refere a controlabilidade medida em que existem formas alternativas de completar. Enquanto reversibilidade se refere a à possibilidade para desfazer ações e retornar para um estado anterior.

Segurança refere-se ao grau em que a tarefa como implementado não causa danos ou riscos, dividido em usuário, terceiros, ambiente e sistema. Usuário se refere a segurança física, legal e confidencial. A Segurança do sistema se refere ao ambiente não causar danos ao sistema.

Critica A medida em que o desempenho da tarefa é decisivo. Divide-se em precisão, robustez e tempo. A precisão refere-se ao nível de precisão exigido na tarefa. Já robustez, a importância da tarefa ser resistente a erros, mesmo em circunstâncias adversas. E tempo, refere-se à velocidade que a tarefa deve ser completada.

Fonte: Mosqueira-Rey Alonso-Ríos e Moret-Bonillo (2009) adaptado pelo autor 
O terceiro e último grupo de atributos definidos por Mosqueira-Rey Alonso-Ríos, e MoretBonillo (2009) é o ambiental, (ver quadro 4) divididos em físicos, sociais e técnicos. Estes atributos ambientais estão apresentados de forma explicativa na tabela a seguir:

Quadro 4 - Dimensões do contexto - Ambiental

Físico Condições São referentes a características do ambiente físico, na qual afeta as percepções do sensoriais usuário. Para este atributo, uma distinção é desenhada entre a qualidade sensorial (condições apropriadas para usar o sistema), esses atributos são descriminados pelos 5 sentidos (visão, audição, tátil, olfativa e gustativa).

\begin{tabular}{ll}
\hline $\begin{array}{l}\text { Condições } \\
\text { atmosféricas }\end{array}$ & Referem-se as características do ar. \\
\hline $\begin{array}{l}\text { Condições } \\
\text { espaciais }\end{array}$ & $\begin{array}{l}\text { São referentes a localização física dentro do ambiente no qual o ambiente é utilizado, } \\
\text { com distinção entre disponibilidade de espaço (tendo espaço necessário para operar o } \\
\text { sistema); aptidão para localização (mais detalhado em equação do local de trabalho e } \\
\text { adequação do sistema); aptidão postural (se o espaço permite que o corpo do usuário se } \\
\text { posicione de forma adequada para utilizar o sistema. }\end{array}$
\end{tabular}

Segurança E segurança refere-se ao grau que o ambiente físico pode causar danos ou riscos, podem ser divididos em atributos como segurança do usuário, acerca da segurança física, legal confidencial e de propriedade e segurança do sistema, ligado a questões que o ambiente pode ou não causar danos ao sistema.

Social Relações de Está associado as interações entre membros da organização dentro da qual o sistema é trabalho usado, este atributo possui os seguintes sub-atributos: equipe de trabalho (interações com outras pessoas durante o processo de uso), suporte humano (se o usuário pode contar com a ajuda de outras pessoas); interrupções (se o trabalho de um usuário pode ser interrompido por outras pessoas).

Objetivos Está correlacionado as intenções da organização em relação as interações de usuários com o sistema.

Controle Envolve a autonomia do usuário em termos de implementar tarefas

Técnico Adequação do Refere-se à medida que o equipamento suporta o funcionamento do sistema e permite equipamento que o sistema seja utilizado.

físico a

usabilidade

Adequação do Está associada à medida que o equipamento (não físico) suporta o funcionamento do equipamento sistema

lógico a

usabilidade

Adequação do Refere-se á medida em que os materiais utilizados durante o funcionamento do sistema material de viáveis a serem utilizados.

consumo a

usabilidade

Fonte: Mosqueira-Rey Alonso-Ríos e Moret-Bonillo (2009) adaptado pelo autor

\section{Resultados}

Uma visão superficial irá tratar o contexto como apenas os fatores do ambiente. Entretanto, como apresentado neste estudo, o conceito de contexto é muito mais amplo. Envolve fatores sociais, da interação do usuário com outras pessoas; do seu estado emocional; fatores da infraestrutura técnica do equipamento ou demais instrumentos envolvidos, entre outros. 
Dessa forma o presente estudo tem como resultado a convergência dos fatores e abordagens listados, com intuito de facilitar o entendimento dos fatores envolvidos no contexto. Assim, a partir do levantamento realizado, foi possível elaborar uma tabela (ver quadro 5) na qual apresenta as abordagens dos diferentes autores, (BELK, 1975; KIM et al., 2005; SCHMIDT, BEIGL, e GELLERSEN, 1999); MOSQUEIRA-REY, ALONSO-RIOS e MORET BONILLO, 2009) suas semelhanças e diferenças.

Quadro 5 - Relações entre os modelos do contexto de uso

\begin{tabular}{|c|c|c|c|c|}
\hline \multicolumn{5}{|c|}{ Modelo do contexto } \\
\hline \multirow[t]{5}{*}{$\begin{array}{l}\text { Contexto } \\
\text { do } \\
\text { Ambiente }\end{array}$} & \multirow[t]{2}{*}{ Físico } & \multirow[t]{2}{*}{$\begin{array}{l}\text { Condições Físicas do } \\
\text { Ambiente/ Físico/ } \\
\text { Localização/ } \\
\text { Condições } \\
\text { sensoriais } \\
\text { Segurança }\end{array}$} & $\begin{array}{l}\text { Barulho, luminosidade, pressão; Ruído; } \\
\text { Localização geográfica, e tempo; }\end{array}$ & $\begin{array}{l}\text { Belk (1975); Kim et al (2005); } \\
\text { Schmidt, Beigl, e Gellersen } \\
\text { (1999); Mosqueira-Rey, Alonso- } \\
\text { Rios e Moret-Bonillo (2009) }\end{array}$ \\
\hline & & & $\begin{array}{l}\text { Grau que o ambiente pode causar } \\
\text { danos ou riscos ao usuário. Podem ser } \\
\text { segurança física, legal, confidencial e } \\
\text { segurança do sistema }\end{array}$ & $\begin{array}{l}\text { Mosqueira-Rey, Alonso-Rios e } \\
\text { Moret-Bonillo (2009) }\end{array}$ \\
\hline & \multirow[t]{2}{*}{ Social } & Social & $\begin{array}{l}\text { Interação interpessoal e a influência de } \\
\text { outras pessoas; }\end{array}$ & $\begin{array}{l}\text { Belk (1975); Kim et al (2005); } \\
\text { Schmidt, Beigl, e Gellersen } \\
\text { (1999) }\end{array}$ \\
\hline & & Temporal & $\begin{array}{l}\text { Ponto no tempo ou o tempo relativo a } \\
\text { algum evento passado ou futuro }\end{array}$ & Belk (1975); \\
\hline & Técnico & $\begin{array}{l}\text { Infraestrutura/ } \\
\text { Adequações do } \\
\text { técnicas equipamento físico, } \\
\text { equico. }\end{array}$ & $\begin{array}{l}\text { Recursos computacionais do ambiente. } \\
\text { Medida que o equipamento suporta } \\
\text { funcionamento do sistema. }\end{array}$ & $\begin{array}{l}\text { Schmidt, Beigl, e Gellersen } \\
\text { (1999); Mosqueira-Rey, Alonso- } \\
\text { Rios e Moret-Bonillo (2009) }\end{array}$ \\
\hline \multirow[t]{4}{*}{$\begin{array}{l}\text { Contexto } \\
\text { Pessoal }\end{array}$} & \multirow[t]{3}{*}{$\begin{array}{l}\text { Interno/ } \\
\text { Usuário }\end{array}$} & Experiência & $\begin{array}{l}\text { Habilidades práticas, conhecimento do } \\
\text { usuário com o sistema }\end{array}$ & $\begin{array}{l}\text { Mosqueira-Rey, Alonso-Rios e } \\
\text { Moret-Bonillo (2009) }\end{array}$ \\
\hline & & Educação & $\begin{array}{l}\text { Educação formal, social, cultural ou } \\
\text { organizacional. Capacidade de } \\
\text { compreender as convenções utilizadas } \\
\text { no sistema }\end{array}$ & $\begin{array}{l}\text { Mosqueira-Rey, Alonso-Rios e } \\
\text { Moret-Bonillo (2009) }\end{array}$ \\
\hline & & $\begin{array}{l}\text { Estado } \\
\text { antecedente/ } \\
\text { Atitude para } \\
\text { sistema }\end{array}$ & $\begin{array}{l}\text { Objetivos e emoção; estado emocional, } \\
\text { condições biofisiológicas; } \\
\text { Consequência do humor do usuário, ou } \\
\text { possíveis mudanças }\end{array}$ & $\begin{array}{l}\text { Kim et al (2005); Schmidt, Beigl, e } \\
\text { Gellersen (1999); Belk (1975); } \\
\text { Mosqueira-Rey, Alonso-Rios e } \\
\text { Moret-Bonillo (2009) }\end{array}$ \\
\hline & \multicolumn{2}{|c|}{$\begin{array}{l}\text { Externo/Usuário/ } \\
\text { Características físicas }\end{array}$} & $\begin{array}{l}\text { Características do corpo que tem } \\
\text { impacto na usabilidade. Características } \\
\text { sensoriais, falar, motora. Limitações ou } \\
\text { aptidões. }\end{array}$ & $\begin{array}{l}\text { Kim et al (2005); Mosqueira-Rey, } \\
\text { Alonso-Rios e Moret Bonillo } \\
\text { (2009) }\end{array}$ \\
\hline \multirow[t]{4}{*}{ Tarefa } & \multicolumn{2}{|c|}{ Tarefa/Função } & $\begin{array}{l}\text { Engajamento na tarefa, objetivos. } \\
\text { Como funciona a interação do usuário } \\
\text { com o sistema, seja em um papel direto } \\
\text { ou indireto }\end{array}$ & $\begin{array}{l}\text { Belk (1975); Schmidt, Beigl, e } \\
\text { Gellersen (1999); Mosqueira- } \\
\text { Rey, Alonso-Rios e Moret Bonillo } \\
\text { (2009) }\end{array}$ \\
\hline & \multicolumn{2}{|c|}{ Uso do sistema } & $\begin{array}{l}\text { Frequência e duração de uso do } \\
\text { sistema. }\end{array}$ & $\begin{array}{l}\text { Mosqueira-Rey, Alonso-Rios e } \\
\text { Moret Bonillo (2009) }\end{array}$ \\
\hline & \multicolumn{2}{|c|}{ Controlabilidade } & $\begin{array}{l}\text { Possibilidade de desfazer ações e } \\
\text { retornar para o estado anterior }\end{array}$ & $\begin{array}{l}\text { Mosqueira-Rey, Alonso-Rios e } \\
\text { Moret Bonillo (2009) }\end{array}$ \\
\hline & \multicolumn{2}{|l|}{ Crítica } & $\begin{array}{l}\text { Precisão exigida para completar a } \\
\text { tarefa e velocidade que a tarefa deve } \\
\text { ser completada }\end{array}$ & $\begin{array}{l}\text { Mosqueira-Rey, Alonso-Rios e } \\
\text { Moret Bonillo (2009) }\end{array}$ \\
\hline
\end{tabular}

Fonte: Criado pelo autor 
Destaca-se que a nomenclatura de alguns fatores encontrados, se diferenciam de acordo com cada autor, contudo, percebeu-se uma similaridade entre os conceitos e a partir desta análise fez-se uma adequação dos mesmos.

Após a análise dos modelos de contexto listados no presente artigo, Belk (1975); Kim et al (2005) e Schmidt, Beigl, e Gellersen (1999); Mosqueira-Rey, Alonso-Rios e Moret Bonillo (2009); foi possível determinar que o contexto de uso para dispositivos móveis contempla principalmente 3 grandes áreas: contexto do ambiente: envolvendo o contexto físico, social e técnico; contexto pessoal: compreende o contexto interno e externo do usuário; e contexto da tarefa: que inclui a tarefa em si entre outros elementos.

\section{Conclusão}

Primeiramente o artigo apresenta as relações entre ergonomia, usabilidade e contexto de uso. A partir do exposto, no que tange a abrangência das áreas, a ergonomia é uma ciência mais abrangente, e esta envolve outros conceitos como usabilidade, experiência do usuário e contexto de uso.

A usabilidade se insere como parte importante da ergonomia, porém tem seu foco mais específico nas relações do usuário na interação com os objetivos, na eficiência e eficácia em utilizálos, assim como na satisfação. Destaca-se que nas definições da ISO 9241-11 (2002), o contexto de uso se insere como parte importante da usabilidade, envolvendo usuários, tarefas, equipamento, o ambiente físico e social no qual o produto é utilizado.

Mesmo que a preocupação esteja sob o foco da usabilidade, quando se trata de dispositivos móveis o contexto de uso é um fator que necessita de devida atenção. Para isto, o presente estudo listou definições do contexto de uso, assim como os fatores envolvidos.

A partir dos resultados apresentados, dos fatores do contexto, fica claro que a experiência do usuário de dispositivos móveis, não está ligada apenas a questões da interface, compreensão e identificação; mas em adição a outros fatores do contexto de uso que interferem diretamente na interação com os dispositivos.

O estudo apresentou uma proposta inicial de convergência dos modelos, afim de facilitar a aplicação e viabilidade para projetos dessa natureza. Para estudos futuros, destaca-se que há a necessidade de um olhar atento nas áreas que envolvem o contexto; o desenvolvimento de um modelo único, amplo, que englobe os fatores listados pelos autores; ou que envolva outros possíveis fatores que interferem no contexto de uso.

\section{Referências}

ALSHEHRI, F. \& FREEMAN, M. (2012). Methods for usability evaluations of mobile devices. In J. W. Lamp (Eds.). 23rd Australian Conference on Information Systems (pp. 1-10). Geelong: Deakin University.

BARNARD, Leon et al. Capturing the effects of context on human performance in mobile computing systems. Personal and Ubiquitous Computing, v. 11, n. 2, p. 81-96, 2007.

BELK, Russell W. Situational variables and consumer behavior. Journal of Consumer research, v. 2, n. 3, p. 157-164, 1975.

BERTINI, Enrico, et al. "Appropriating heuristic evaluation for mobile computing." International 
Journal of Mobile Human Computer Interaction (IJMHCI) 1.1 (2009): 20-41.

BEVAN, N. Workshop: Meaningful Measures: Valid Useful User Experience Measurement. Classifying and selecting UX and usability measures. Interactions 13, 2008.

CAÑAS, J.J. WAERS, Y.Ergonomia Cognitiva - Aspectos psicológicos de la Interacción de las Personas con la Tecnologia de la Información. Ed. Medica Panamericana. 2001.

CYBIS, Walter de Abreu; BETIOL, Adriana Holtz; FAUST, Richard. Ergonomia e usabilidade: conhecimentos, métodos e aplicações. 2. ed. rev. e ampl. São Paulo (SP): Novatec, 2010. 422 p. ISBN 9788575222324.

CYBIS, Walter de Abreu; BETIOL, Adriana Holtz; FAUST, Richard. Ergonomia e usabilidade: conhecimentos, métodos e aplicações. 2. ed. rev. e ampl. São Paulo (SP): Novatec, 2010. 422 p.

IIDA, I. Ergonomia: Projeto e Produção. São Paulo: Edgard Blücher, 1990.

INTERNATIONAL ORGANIZATION FOR STANDARDIZATION. ISO 9241-11: Ergonomic requirements for office work with visual display terminals (VDTs) - pt.11: guindance on usability. Geneva: ISO, 1998. IV,22p.

ISO/IEC. 2000. ISO 9241-9:2000: Ergonomic requirements for office work with visual display terminals (VDTs) -- Part 9: Requirements for non-keyboard input devices. Geneva: ISO, 1998. IV,22p.

ISO/IEC. 2001. ISO 9241-11: Ergonomic requirements for office work with visual display terminals (VDTs) - pt.11: guindance on usability. Geneva: ISO, 1998. IV,22p.

ISO/IEC. 2001. ISO 9241-11: Ergonomic requirements for office work with visual display terminals (VDTs) - pt.11: guindance on usability. Geneva: ISO, 1998. IV,22p.

KIM, H., Kim, J., Lee, Y. 2005, An Empirical Study of Use Contexts in the Mobile Internet, Focusing on the Usability of Information Architecture. Information Systems Frontiers 7:2, 175-186.

LAKATOS, E.M. Metodologia Científica. 1 ed. São Paulo. Atlas, 1983. 231p.

LIMA, S.L.S. Ergonomia cognitiva e a interação pessoa-computador: analise da usabilidade da urna eletrônica 2002 e do modulo impressor externo. 2003. Dissertação (mestrado em Engenharia de produção) - Universidade Federal de Santa Catarina.

MAGUIRE, M. Context of use within usability activities. International Journal of Human-Computer Studies, v. 55, n. 4, p. 453-483, Oct 2001. ISSN 1071-5819

MALLAT, Niina et al. The impact of use context on mobile services acceptance: The case of mobile ticketing. Information \& management, v. 46, n. 3, p. 190-195, 2009.

MORAIS, Anna Beatriz Dias; SEABRA, Rodrigo Duarte; MATTEDI, Adriana Prest. Um Estudo sobre a Usabilidade do Facebook em Dispositivos Móveis com Ênfase em Usuários Idosos. Anais do Computer on the Beach, p. 198-207, 2018.

MORAES, A. (1996) Et al. "Ergonomia, usabilidade e qualidade de produtos: conforto e segurança dos usuários; defesa do consumidor." In Anais P\&D Design. Belo Horizonte.

MORAES, Ana Maria de. Design e avaliação de interface: ergo design e interação humanocomputador. Rio de Janeiro: iUsEr, 2002. 147 p.: ISBN 8590286215. 
MORAES, Ana Maria; Mont'alvão, Claudia. Ergonomia: conceitos e aplicações. 2a edição. Editora 2AB. Rio de Janeiro. 2000.

MOSQUEIRA-REY, Eduardo; ALONSO-RÍOS, David; MORET-BONILLO, Vicente. Usability taxonomy and context-of-use taxonomy for usability analysis. In: Systems, Man and Cybernetics, 2009. SMC 2009. IEEE International Conference on. IEEE, 2009. p. 812-817.

PEIXOTO, José Rui da Costa Alves. Mobile context awareness: estado da arte e casos práticos. 2017. Dissertação de Mestrado.

PREZOTTO, Ezequiel Douglas; BONIATI, Bruno Batista. Estudo de frameworks multiplataforma para desenvolvimento de aplicações mobile híbridas. 2017.

REMUSZKA, Anderson Jose; OLIVEIRA, Aurélio Luiz. A IMPORTANCIA DA DISCIPLINA DE ERGONOMIA PARA ACADÊMICOS DO CURSO DE BACHARELADO EM EDUCAÇÃO FÍSICA. Anais da Jornada Científica dos Campos Gerais, v. 15, 2017.

ROTO, Virpi et al. Web browsing on mobile phones: Characteristics of user experience. Helsinki University of Technology, 2006.

SCHMIDT, Albrecht; BEIGL, Michael; GELLERSEN, Hans-W. There is more to context than location. Computers \& Graphics, v. 23, n. 6, p. 893-901, 1999.

SEFFAH, Ahmed et al. Usability measurement and metrics: A consolidated model. Software Quality Journal, v. 14, n. 2, p. 159-178, 2006.

SHACKEL, B. Ergonomics in design for usability. People and computers: Designing for usability. pp.44-64. Proceedings of $\mathrm{HCl} 86$. Cambridge, UK, 1986.

TE'ENI, D., CAREY, J. and ZHANG, P. 2007. Human computer interaction: developing effective organizational information systems New York: Wiley.

WISNER, A. Por dentro do trabalho: Ergonomia, método \& técnica. São Paulo: FTD: Oboré, 1987.

Zuehlke, D., \& Thiels, N. (2008). Useware engineering: a methodology for the development of userfriendly interfaces. Library Hi Tech, 26(1), 126-140. 